\title{
VÍDEO-HISTEROSCOPIA DA IMAGEM ENDOMETRIAL ALTERADA: UMA AVALIAÇÃO CRÍTICA
}

\author{
HYSTEROSCOPY WITH ABNORMAL ENDOMETRIAL IMAGE: CRITICAL \\ EVALUATION
}

Laura Osthoff, TCBC-RJ1'; Hilton Augusto Koch²; Alkindar Soares ${ }^{3}$

\begin{abstract}
RESUMO: Objetivo: Analisar a concordância da imagem endometrial pela vídeo-histeroscopia e pela ultra-sonografia Método: Análise retrospectiva de 1561 laudos endoscópicos no período de 1999 a 2005. Foi avaliada a acurácia das imagens video-histeroscópicas em relação à imagens ultra-sonográficas do endométrio alterado com avaliação dicotomizada em imagens benignas e complexas. Foram também analisadas por período do menacme e pós-menopausa. Resultados: Foi encontrada uma maior incidência de hipertrofias benignas: pólipos- 58,7\%; hipertrofias simples - 13,3\%; endometrites $3,5 \%$; miomatose $-6,0 \%$; outros $-11,9 \%$ : cavidade sem alterações $0,9 \%$ : baixa incidência de hipertrofias complexas no menacme e 5,3 vezes maior na pós- menopausa. Conclusão: Há concordância entre as variáveis ultra-sonográficas do endométrio alterado com o diagnóstico histeroscópico (Rev. Col. Bras. Cir. 2007; 34(6): 401-406).
\end{abstract}

Descritores: Endométrio; Ultra-sonografia; Histeroscopia; Neoplasias do endométrio.

\section{INTRODUÇÃO}

A comparação das imagens ultra-sonográficas do endométrio espessado com as imagens vídeo histeroscópicas é utilizada como padrão ouro ${ }^{1}$ para o diagnóstico em doenças do endométrio.

O câncer endometrial se constitui em uma doença que tende a aumentar com a maior longevidade das mulheres, considerando-se o contexto das modificações comportamentais da mulher na atualidade, com as conseqüentes alterações na sua saúde².

O diagnóstico precoce das lesões precursoras do câncer do endométrio pode ser realizado através da histeroscopia que normalmente é precedida pelas imagens ultra-sonográficas da cavidade uterina ${ }^{3}$.

O presente estudo tem como objetivo avaliar a importância da comparação entre as imagens vídeo histeroscópica e ultra-sonográficas para a definição e complementação das alterações endometriais.

\section{MÉTODO}

Trata-se de estudo retrospectivo, em que os laudos das pacientes com endométrio alterado na ultra-sonografia foram avaliados pelos exames vídeo histeroscópicos.

O estudo foi realizado no Ambulatório de Ginecologia da $28^{\mathrm{a}}$. Enfermaria da Santa Casa da Misericórdia do Rio de Janeiro, Setor de Endoscopia Ginecológica. Este Hospital Geral recebe pacientes de vários Centros Ultrasonográficos e de diferentes Centros de Atendimento à Mulher, obedecendo à lógica do sistema de Referência e Contra-referência ${ }^{4}$, que é o processo de articulação com outras instituições de saúde para o encaminhamento, transferência e recepção (contra-referência) de pacientes. Ao chegar em nosso ambulatório a paciente apresenta o laudo ultrasonográfico do centro de atendimento e o pedido de exame de histeroscopia. Após a realização do exame a paciente retorna com o laudo padronizado ao seu centro de atendimento.

A amostra foi homogeneizada e os exames histeroscópicos foram realizados unicamente pela autora do artigo.

Foi realizada a anamnese ginecológica e avaliada a solicitação do exame histeroscópico. Os dados foram inseridos em laudo sistematizado.

Após a colocação do espéculo, inicia-se a aproximação do colo uterino através do orifício externo passando-se a estudar cada segmento do canal cervical, orifício interno e cavidade uterina. Essas imagens são avaliadas através de um monitor de alta resolução. Após o exame, os laudos são completados com os achados endoscópicos, e é sugerido o diagnóstico. Para algumas pacientes são solicitadas avaliações histopatológica através da biópsia dirigida, de acordo com o pedido do encaminhamento para o exame endoscópico.

\footnotetext{
1. Responsável pelo Serviço de Endoscopia Ginecológica da Santa Casa da Misericórdia do Rio de Janeiro - RJ.

2. Professor Titular de Radiologia da Universidade Federal do Rio de Janeiro; Chefe do Serviço de Radiologia da Santa Casa da Misericórdia do Rio de Janeiro- RJ.

3. Chefe do Serviço de Ginecologia da Santa Casa da Misericórdia do Rio de Janeiro - RJ.

Recebido em 15/08/2007
}

Aceito para publicação em 28/09/2007

Conflito de Interesses: nenhum

Fonte de financiamento: nenhuma

Trabalho realizado na Santa Casa da Misericórdia do Rio de Janeiro, Serviço de Radiologia e 28 Enfermaria, Serviço de Ginecologia. 
Foram avaliados 1561 laudos emitidos entre março de 1999 e dezembro de 2005. Foi organizada uma tabela para a identificação dos diagnósticos histeroscópicos e das solicitações dos exames.

Foram selecionados os laudos das pacientes nas quais a indicação para os exames histeroscópicos eram as alterações ultra-sonográficas no endométrio. Foram identificados 761 laudos. Estes foram comparados com os respectivos diagnósticos histeroscópicos.

Foram excluídos 21 laudos de toda a planilha, de pacientes impedidas para o exame histeroscópico: estenose fibrosa do orifício interno (OI), reação álgica, infecção aguda e os laudos que não estavam completos.

Os critérios ultra-sonográficos utilizados para a caracterização das imagens endometriais foram baseados da medida da camada basal da parede anterior à posterior, no corte longitudinal, considerando: a) Diâmetro acima de $5 \mathrm{~mm}$ na pós-menopausa sem terapia de reposição hormonal (TH). Podemos encontrar imagens de calcificação, imagens de cistos, irregularidades e espessamentos polipóides; b) Espessura acima de $14 \mathrm{~mm}$ no menacme; c) Líquido na cavidade; d) Heterogeneidade

De acordo com as imagens histeroscópicas, foram caracterizados dois grupos: a) Imagens benignas: São imagens que estão em acordo com a faixa etária e a data do ciclo menstrual. Hipertrofias simples; hipertrofias polipóides; endometrites; pólipos; adenomiose; sinéquias; atrofia; dispositivo intra-uterino (DIU) em cavidade; distrofias vasculares; septos; mioma submucoso e b) Imagens Suspeitas (natureza complexa): São imagens que mostram alteração na formação dos vasos, da superfície da cor e sugerem início de processo de degeneração (necrose - sangramento). Hipertrofias císticas; hematométria; hipertrofias cerebróides; atrofia cística.

Foram considerados resultados em concordância positiva, quando ambos os métodos foram anormais, ou seja, as imagens encontradas eram sugestivas de patologias endometriais.

Foram considerados resultados em concordância negativa quando estavam em desacordo. A ultra-sonografia estava alterada e o exame histeroscópico revelava cavidade uterina sem alteração.

Foram classificados também por faixa etária: laudos de pacientes no menacme e laudos de pacientes na pós- menopausa
Estes resultados foram analisados de forma dicotomizada, normal e anormal. Segundo os critérios de Labastida $^{5}$ (Universidade de Barcelona) as espessuras endometriais encontradas de acordo com a data do ciclo menstrual, regenerativo, proliferativo, secretor, pré-menstrual e menstrual são normais, no menacme. Os atróficos (no menacme), assincrônicos, hipertrofias simples, polipóides e complexas, são considerados anormais tanto no menacme como na pós-menopausa.

\section{RESULTADOS}

Dos 1561 exames (607 na menopausa e 954 no menacme), foram selecionados os laudos que apresentavam como solicitação a ultra-sonografia com espessamento do endométrio. Foram identificadas 761 pacientes com este pedido, (48,7\%) - A idade variou de 16 aos 83 anos. Destas, 428 eram da menopausa (56.2\%), última menstruação acima de um ano e as demais 333 no menacme $(43,7 \%)$.

De toda a planilha analisada, a maior incidência foi na $5^{\mathrm{a}}$ década da vida, com 380 pacientes $(24,2 \%)$ (Tabela 1$)$.

Ao se relacionar os achados benignos (Tabela 2) encontramos predomínio acentuado quanto à freqüência de pólipos $(56,0 \%)$ e baixa incidência de cavidade sem doenças $(0.9 \%)$.

Ao se relacionar às imagens complexas no menacme observa-se cinco pacientes com adenocarcinoma, pólipos degenerados e metaplasia (1,6\%),

Na tabela 3, são apresentados os dados das imagens benignas relativos aos diagnósticos no período da pós-menopausa.

No detalhamento da ocorrência "outros", foram identificadas as seguintes imagens histeroscópicas: adenomiose, anel de estenose, DIU perdido, metaplasia óssea, mola hidatiforme, muco, obstrução tubária e sinéquias.

Houve um predomínio acentuado quanto à freqüência de pólipos. Houve baixa incidência de cavidade sem alterações $1 \%$

Ao se relacionar as Imagens complexas na pós-menopausa os dados obtidos apontam para 39 casos, que correspondem a $8,51 \%$ dos laudos analisados. Todas as imagens complexas foram sugestivas de adenocarcinoma (Tabela 4). Com estes resultados temos como desdobramento: 754 laudos com concordância positiva $(99,08 \%)$ e sete com concordância negativa $(0,9 \%)$.

Tabela 1 - Distribuição de freqüências dos laudos por década de vida.

\begin{tabular}{rccccccccc}
\hline & $\mathbf{2}^{\text {a }}$ Década & $\mathbf{3}^{\text {a }}$ Década & $\mathbf{4}^{\mathbf{a}}$ Década & $\mathbf{5}^{\text {a }}$ Década & $\mathbf{6}^{\mathbf{a}}$ Década & $\mathbf{7}^{\mathbf{a}}$ Década & $\mathbf{8}^{\mathbf{a}}$ Década & $\mathbf{9}^{\mathbf{a}}$ Década & Total \\
\hline 1999 & 3 & 18 & 42 & 21 & 20 & 8 & 4 & 1 & 117 \\
2000 & 3 & 23 & 31 & 26 & 12 & 15 & 4 & 1 & 115 \\
2001 & 2 & 26 & 49 & 50 & 36 & 19 & 20 & 5 & 207 \\
2002 & 3 & 13 & 32 & 37 & 30 & 26 & 13 & 1 & 155 \\
2003 & 3 & 18 & 38 & 70 & 48 & 31 & 20 & 1 & 229 \\
2004 & - & 21 & 71 & 93 & 77 & 70 & 35 & 6 & 373 \\
2005 & - & 41 & 68 & 83 & 75 & 64 & 32 & 2 & 365 \\
Total & 14 & 160 & 331 & 380 & 298 & 233 & 128 & 17 & 1561 \\
\hline
\end{tabular}


Tabela 2 - Achados ðiagnósticos Benignos no menacme em cada período estudado.

\begin{tabular}{lrrrrrrrrr}
\hline DIAGNÓSTICOS & $\mathbf{1 9 9 9}$ & $\mathbf{2 0 0 0}$ & $\mathbf{2 0 0 1}$ & $\mathbf{2 0 0 2}$ & $\mathbf{2 0 0 3}$ & $\mathbf{2 0 0 4}$ & $\mathbf{2 0 0 5}$ & Total & \% \\
\hline Pólipos & - & 5 & 21 & 25 & 39 & 47 & 51 & 188 & 56.0 \\
Hipetrofias Simples & - & 2 & 12 & 10 & 14 & 20 & 14 & 72 & 22.0 \\
Endometrite & - & - & 2 & 2 & 3 & 1 & 3 & 11 & 3,5 \\
Miomas & - & - & 5 & 5 & 6 & 3 & 10 & 29 & 8,4 \\
Outros & - & 2 & 3 & 3 & 14 & 6 & 4 & 32 & 9,2 \\
Cavidade sem alterações & - & - & 3 & - & - & - & - & 3 & 0,9 \\
Total & - & 9 & 46 & 45 & 76 & 77 & 82 & 335 & 100 \\
\hline
\end{tabular}

Tabela 3 - Imagens Benignas na pós- menopausa.

\begin{tabular}{lrrrrrrrrr}
\hline Diagnóstico & $\mathbf{1 9 9 9}$ & $\mathbf{2 0 0 0}$ & $\mathbf{2 0 0 1}$ & $\mathbf{2 0 0 2}$ & $\mathbf{2 0 0 3}$ & $\mathbf{2 0 0 4}$ & $\mathbf{2 0 0 5}$ & Total & \% \\
\hline Pólipos & 3 & 7 & 27 & 32 & 51 & 74 & 62 & 256 & $\mathbf{6 5 , 8}$ \\
Hipertrofia simples & - & 1 & 5 & 12 & 2 & 5 & 8 & 33 & $\mathbf{8 , 4}$ \\
Endometrite & - & - & 4 & - & 3 & 5 & 4 & 16 & $\mathbf{4 , 1}$ \\
Miomatose & 1 & 1 & 4 & - & 3 & 3 & 7 & 19 & $\mathbf{4 , 8}$ \\
Outros & 3 & 2 & 3 & 10 & 16 & 14 & 13 & 61 & $\mathbf{1 5 , 6}$ \\
Cavidade sem alterações & 1 & - & - & 1 & - & 2 & - & 4 & $\mathbf{1 , 0}$ \\
Total & 8 & 11 & 43 & 55 & 75 & 103 & 94 & 389 & $\mathbf{1 0 0 , 0}$ \\
\hline
\end{tabular}

Na tabela 5 observa-se as associações de variáveis no período menarca , pós-menopausa e total da amostra, teste não-paramétrico de $\mathrm{X}^{2}$ (qui-quadrado) e sua significância para os anos de 1999 a 2005.

A análise dos dados mostrou que dos 1561 laudos avaliados, 761 tinham alteração no ultra-som e apenas 21 não foram conclusivos; as imagens histeroscópicas trazem noções de volume, textura, superfície, vascularização, precisa localização, gerando com isso melhor analise e, portanto decisões mais adequadas; as imagens ultra-sonográficas são bem definidas pelo estudo histeroscópico realizado ambulatorialmente.

\section{DISCUSSÃO}

Os avanços tecnológicos absorvidos na Medicina têm absoluta influência nos exames de imagem. Pode-se dividir o estudo da pelve em antes e após a ultra-sonografia. Estes equipamentos trazem melhoria progressiva da definição das estruturas. Porém, em relação a cavidade uterina a descrição da doença se mostra limitada.

Os avanços que têm sido incorporados à histeroscopia na última década têm permitido maior segurança no procedimento, além da obtenção de imagens mais nítidas na suspeição diagnóstica, o que tem melhorado os resultados oriundos das biópsias endometriais. A histeroscopia está indicada em todas as situações clínicas nas quais a visualização da cavidade uterina possa fornecer subsídios para um diagnóstico preciso e conseqüente conduta terapêutica. A avaliação do sangramento uterino anormal e a investigação do fator uterino na infertilidade constituem as indicações mais freqüentes para a realização da histeroscopia ${ }^{6}$.
Tabela 4 - Imagens complexas na menopausa.

\begin{tabular}{rr}
\hline Anos & Freqüiências \\
\hline 2000 & 3 \\
2001 & 9 \\
2002 & 5 \\
2003 & 7 \\
2004 & 4 \\
2005 & 11 \\
Total & 39 \\
\hline
\end{tabular}

Na pesquisa do sangramento uterino é fundamental diferenciar as pacientes pré e pós-menopausadas. Nessas etapas da vida da mulher, as causas determinantes do sangramento variam, principalmente, em função dos níveis séricos dos hormônios esteróides ovarianos. Na pré-menopausa, em aproximadamente $50 \%$ das pacientes, a etiologia é funcional. Na pós-menopausa o prognóstico é mais grave e as causas residem, na maioria das vezes, numa doença orgânica, sobressaindo-se os pólipos, os miomas submucosos, hiperplasias endometriais e, mais raramente, o carcinoma do endométrio. Neste grupo de mulheres, o procedimento histeroscópico deve ser sempre complementado com a retirada de fragmento tecidual para a realização de exame histopatológico ${ }^{7}$.

A alta ocorrência de carcinoma endometrial em mulheres com sangramento pós-menopausa reforça a necessidade de um procedimento diagnóstico mais acurado ${ }^{8}$.

Neste estudo foram avaliados apenas os laudos das pacientes com a indicação de alteração do endométrio à ultra- 
Tabela 5 - Associações de variáveis no período menarca, pós-menopausa e total da amostra, teste não-paramétrico de $X^{2}$ (quiquadrado) e sua significância.

\begin{tabular}{|c|c|c|c|}
\hline Ano/Período & Associações & $\mathbf{X}^{2}$ & Significância \\
\hline 1999/Menarca & Indicação X Histeroscopia & 81,01 & $(\mathrm{P}=0,001)^{* *}$ \\
\hline 1999/Pós-Menopausa & Indicação X Histeroscopia & 50,78 & $(\mathrm{P}=0,001)^{* *}$ \\
\hline 1999/Total & Indicação X Histeroscopia & 102,31 & $(\mathrm{P}=0,001)^{* *}$ \\
\hline 2000/Menarca & Indicação X Histeroscopia & 198,68 & $(\mathrm{P}=0,001)^{* *}$ \\
\hline 2000/Pós-Menopausa & Indicação X Histeroscopia & 29,87 & $(\mathrm{P}=0,714) n . s$ \\
\hline 2000/Total & Indicação X Histeroscopia & 191,14 & $(\mathrm{P}=0,001)^{* *}$ \\
\hline 2001/Menarca & Indicação X Histeroscopia & 122,30 & $(\mathrm{P}=0,001)^{* *}$ \\
\hline 2001/Pós-Menopausa & Indicação X Histeroscopia & 26,06 & $(\mathrm{P}=0,985)$ n.s \\
\hline 2001/Total & Indicação X Histeroscopia & 156,85 & $(\mathrm{P}=0,001)^{* *}$ \\
\hline 2002/Menarca & Indicação X Histeroscopia & 75,62 & $(\mathrm{P}=0,001)^{* *}$ \\
\hline 2002/Pós-Menopausa & Indicação X Histeroscopia & 88,42 & $(\mathrm{P}=0,001)^{* *}$ \\
\hline 2002/Total & Indicação X Histeroscopia & 96,45 & $(\mathrm{P}=0,001)^{* *}$ \\
\hline 2003/Menarca & Indicação X Histeroscopia & 88,56 & $(\mathrm{P}=0,001)^{* *}$ \\
\hline 2003/Pós-Menopausa & Indicação X Histeroscopia & 47,93 & $(\mathrm{P}=0,001)^{* *}$ \\
\hline 2003/Total & Indicação X Histeroscopia & 134,50 & $(\mathrm{P}=0,001)^{* *}$ \\
\hline 2004/ Menarca & Indicação X Histeroscopia & 125,83 & $(\mathrm{P}=0,001)^{* *}$ \\
\hline 2004/ Pós-enopausa & Indicação X Histeroscopia & 111,71 & $(\mathrm{P}=0,001)^{* *}$ \\
\hline 2004/ Total & Indicação X Histeroscopia & 138,73 & $(\mathrm{P}=0,001)^{* *}$ \\
\hline 2005/Menarca & Indicação X Histeroscopia & 240,03 & $(\mathrm{P}=0,001)^{* *}$ \\
\hline 2005/Pós-Menopausa & Indicação X Histeroscopia & 164,28 & $(\mathrm{P}=0,001)^{* *}$ \\
\hline 2005/Total & Indicação X Histeroscopia & 303,62 & $(\mathrm{P}=0,001)^{* *}$ \\
\hline
\end{tabular}

sonografia, isto é, pacientes no menacme e menopausa que não apresentavam sangramento irregular.

A histeroscopia associada à exérese dirigida de endométrio é rápida, segura e com baixa incidência de complicações, sendo indicado principalmente nos casos de processos focais do endométrio como os pólipos e miomas submucosos 9 .

As ultra-sonografias avaliadas e comparadas neste estudo são das mais diversas procedências. E utilizou-se a medida padrão da camada basal da parte anterior à posterior do útero de acima de $5 \mathrm{~mm}$ para menopausa e $14 \mathrm{~mm}$ para o menacme.

Pólipos e miomas podem, eventualmente, não ser diagnosticados pelos exames tradicionais (curetagem uterina e ultra-sonografia transvaginal) e constituir causa de sangramento recorrente ${ }^{10}$. Nesse estudo a histeroscopia mostrou grande eficiência no diagnóstico de pólipos (59.1\%), concordando com dados da literatura ${ }^{10}$.

Ao se filtrar as variáveis mais importantes, levou-se em consideração o aumento do número de solicitações dos exames endoscópicos para as mulheres que tinham laudos ultra-sonográficos com alterações endometriais.

Na comparação com outros estudos ${ }^{11,12}$ observou-se que o clínico usa critérios sedimentados para caracterizar as pacientes de risco. Porém, vale salientar que um número não desprezível de pacientes com "angústia de saber como está tudo", pressiona o profissional para solicitar exames de rastreio sem indicações precisas.

As dificuldades para a definição da imagem no exame endoscópico se deram em virtude de grande quantidade de muco ou sangue, e quando sanadas, foi solicitado o retorno das pacientes para reexame em 15 dias.

No período de avaliação deste estudo, que se iniciou em 1999 com a implantação e desenvolvimento do Serviço de Endoscopia Ginecológica, observou-se uma diminuição de $70 \%$ das curetagens semióticas no Serviço de Ginecologia da Santa Casa da Misericórdia do Rio de Janeiro e de $47 \%$ em todo o Hospital Geral, segundo dados do Data SUS. Esta constatação tem como reflexo a diminuição de custos hospitalares e de limitação das atividades laborativas das pacientes.

Do ponto de vista da aceitação da comunidade médica, pode-se inferir uma confiança nos resultados obtidos. Nesta base de dados observou-se um contínuo aumento das solicitações dos exames com base na ultra-sonografia alterada no endométrio

A histeroscospia apresenta alta acurácia diagnóstica nos casos de câncer de endométrio ${ }^{13}$ e combinada com a biópsia é citada como "padrão-ouro" no diagnóstico de câncer de endométrio, com $100 \%$ de sensibilidade ${ }^{14}$. Souza et al ${ }^{15}$ em 88 histeroscopias, tiveram nove casos de câncer (13\%), com níveis de sensibilidade de $88,9 \%$, especificidade de $98,3 \%$, valor preditivo positivo de $88,9 \%$ e valor preditivo negativo de $98,3 \%$.

A maioria dos carcinomas do endométrio é representada pelo adenocarcinoma. Outros tipos histológicos incluem o adenoacantoma, o carcinoma de células claras, o carcinoma papilar seroso, etc. Com base na diferenciação histológica são subdivididos em Grau 1 (bem diferenciados), Grau 2 (moderadamente diferenciados) e Grau 3 (pouco diferenciados). Quan- 
to mais indiferenciado for o tumor, mais rápido o seu desenvolvimento e pior o prognóstico ${ }^{16}$.

De acordo com a visão acadêmica tradicional, a hiperplasia do endométrio precede ao carcinoma. O dado mais importante, contudo, não é o tipo da hiperplasia, simples ou complexa, mas sim a presença ou ausência de atipia celular ${ }^{17}$.

Em virtude da dificuldade em solicitar exame histopatológico a todas as pacientes (dificuldades técnicas do Hospital Geral), orientamos, oportunamente, no laudo histeroscópico, sobre a necessidade do prosseguimento da investigação, ou seja, quando avaliamos alterações de natureza complexa, as quais apresentaram baixa incidência nas pacientes do menacme $-1,6 \%$ e de incidência cinco vezes maior na faixa da menopausa $-8,5 \%$.

Embora a maioria dos adenocarcinomas do endométrio seja hormônio-dependente (precedidos por hiperplasia), eles podem se desenvolver de um endométrio atrófico (não-hormônio-dependente). Ocorrem geralmente na pós-menopausa tardia e apresentam um pior prognóstico ${ }^{17}$.

O sintoma mais freqüente da hiperplasia e do adenocarcinoma é o sangramento uterino anormal. Existem, contudo, casos de hiperplasias e mesmo adenocarcinomas "silenciosos" que não se acompanham de sangramento, fato que destaca o teste do progestogênio como uma poderosa e indispensável arma propedêutica no rastreamento dessas lesões $^{17,18}$.

Nesta casuística pode-se observar significância estatística quando comparamos o estudo da cavidade uterina com histeroscopia, em todo o período estudado tanto no menacme quanto na pós-menopausa. São informações que demonstram a oportunidade de rastreio de doenças na cavidade uterina que anteriormente ficavam com o diagnóstico de “alterações endometriais" (Tabela 5).

Quando comparados os diagnósticos ultrasonográficos com os endoscópicos, identificou-se um percentual de discordância muito baixo: apenas $0,91 \%$ dos laudos foram diagnosticados como normais enquanto o ultrasom estava alterado. Sendo o exame ultra-sonográfico, examinador dependente, pode-se inferir, no presente estudo, que as alterações endometriais são bem identificadas pelos profissionais, e que estes estão qualificados para isto.

A correlação das imagens do endométrio alterado à ultra-sonografia com a correspondente imagem histeroscópica, no Hospital Geral da Santa Casa da Misericórdia do Rio de Janeiro revelou uma maior incidência de doenças benignas sendo baixa a incidência de hipertrofias complexas no menacme e 5,3 vezes maior na menopausa. O Grau de acerto entre os dois métodos estudados é significativo, uma vez que apenas 0,9\% dos laudos com ultra-sonografia alterada no endométrio, foi diagnosticado sem alterações na histeroscopia no período do menacme e, 1,0\% na menopausa. Cabe observar que a definição da imagem em pólipo; hipertrofia simples ou complexa, só foi possível na avaliação endoscópica.

Baseados neste estudo, que evidenciou a concordância entre as variáveis ultra-sonográficas do endométrio alterado com o diagnóstico histeroscópico, os autores sugerem a realização da ultra-sonografia trans-vaginal como rastreio, e a histeroscopia para as alterações encontradas ou para as pacientes sintomáticas. A indicação do exame endoscópico como rastreio deve ser mais estudada.

\begin{abstract}
Background: To analyze the incidence of alterations in a comparative study between hysteroscopy and ultrasonography results with abnormal endometrium. Methods: This is a retrospectively reviewed study with 1561 endoscopy reports in the period from 1999 through 2005. It was conducted in the Saint Marries of the Mercy's House of Rio de Janeiro. The accuracy of the hysteroscopy images was evaluated in comparison to the ultrasonography images in patients with abnormal endometrium and was divided between benign and complex images. The fertility period and menopause were also analyzed and considered. Results: The Qui-square study revealed a significant statistical meaning for comparison of the variables: ultrasound with abnormal endometrium versus hysteroscopy for the whole studied period. Conclusion: A large incidence of benign hypertrophies was found: polyps 58,7\%; - simple hypertrophies - 13,3\%; - endometrium with inflammation -3,5\%; fibromas -6,0\%: other11,9\%: cavity without alterations $0,9 \%$ : A low incidence of complex hypertrophies was encountered during the fertility period in comparison with 5.3 times more complex hypertrophies found during the menopause.
\end{abstract}

Key Words: Endometrium; Ultrasonography; Hysteroscopy; Endometrial neoplasms.

\section{REFERÊNCIAS}

1. Widrich T, Bradley LD, Mitchinson AR, Collins RL. Comparison of saline infusion sonography with office hysteroscopy for the evaluation of endometrium. Am J Obstet Gynecol. 1996 Apr;174(4):1327-34.

2. Prota, FE. Avaliação da cavidade uterina através da histeroscopia biópsia endometrial [Dissertação]. UNICAMP, 2001.

3. Mencaglia L. Histeroscopia Cirúrgica. São Paulo: Medsi, 2004

4. ANVISA. Manual Brasileiro de Acreditação. 2002. Disponível em: www.anvisa.gov.br/servicosaude/manuais/mba nefro cliente. Acesso : 1/12/2007.

5. Labastida, R. Tratado y atlas de histeroscopia. Barcelona: Salvat Editores, 1990.

6. Machado MK, Pina H, Matos E. Acurácia da histeroscopia na avaliação da cavidade uterina em pacientes com sangramento uterino pós-menopausa. Rev Bras Ginecol Obstet. 2003;25(4):237-41.

7. Machado, M et al. Histeroscopia. Rev. Bras. Ginecol. Obstet. 2003;25:1-4. 
8. Grimes DA. Diagnostic dilatation and curettage: a reappraisal. Am J Obstet Gynecol. 1982;142(1):1-6.

9. Lasmar R, Barrozo P. Histeroscopia: uma abordagem prática. $1^{\mathrm{a}}$ ed. Rio de Janeiro: Medsi; 2002. p.17-179.

10. Giusa-Chiferi MG, Gonçalves WJ, Baracat EG, Albuquerque Neto LC, Bortoletto CC, Lima GR. Transvaginal ultrasound, uterine biopsy and hysteroscopy for post-menopausal bleeding. Int $\mathrm{J}$ Gynecol Obstet. 1996; 55(1):39-44.

11. Viscomi FA, Lima SM, Aldrighi JM, Ihlenfeld MFK. Freqüência de adenocarcinoma de endométrio em ambulatório de histeroscopia: Um estudo multicêntrico. Rev Bras Ginecol Obstet. 2002;24(1):45-50 .

12. Scavuzzi A, Amorim M, Pinho Neto JS, Santos LC. Comparação entre os achados ultra-sonográficos, histeroscópicos e histopatológicos no sangramento uterino da pós-menopausa. Rev Bras Ginecol Obstet. 2003;25(4):229-35.

13. Hamou J. Hysteroscopy and microhysteroscopy. $1^{\text {st }}$ ed. Norwalk: Appleton \& Lange; 1991.

14. Mortakis AE, Mavrelos K. Transvaginal ultrasonography and hysteroscopy in the diagnosis of endometrial abnormalities. J Am Assoc Gynecol Laparosc. 1997;4(4):449-52.

15. Souza R, Silvestre M, Almeida e Sousa L, Falcão F, Dias I, Silva T, De Oliveira C, Oliveira HM. Transvaginal ultrasonography and hysteroscopy in postmenopausal bleeding: a prospective study. Acta Obstet Gynecol Scand. 2001;80(9):856-62.
16. van Leeuwen FE, Rookus MA. The role of exogenous hormones in the epidemiology of breast, ovarian and endometrial cancer. Eur J Cancer Clin Oncol. 1989;25(12):1961-72.

17. Hulka BS, Fowler WC Jr, Kaufman DG, Grimson RC, Greenberg BG, Hogue CJ, Berger GS, Pulliam CC. Estrogen and endometrial cancer: cases and two control groups from North Carolina. Am J Obstet Gynecol. 1980;137(1):92-101.

18. Gitsch G, Hanzal E, Jensen D, Hacker NF. Endometrial cancer in premenopausal women 45 years and younger. Obstet Gynecol. 1995;85(4):504-8.

Como citar este artigo:

Osthoff L, Koch HA, Soares A. Vídeo-histeroscopia da imagem endometrial alterada: uma avaliação crítica. Rev Col Bras Cir. [periódico na Internet] 2007; 34(6). Disponível em URL: http:// www.scielo.br/rcbc

Endereço para correspondência:

Laura Osthoff

Rua Conde de Irajá, 513 - Botafogo

22271-020 - Rio de Janeiro - RJ

E-mail: laura.osthoff@uol.com.br

Tel: (21) 2539-0537 